\title{
LEISHMANIOSE CUTÂNEA EM COBAIAS (Cavia porcellus)
}

\author{
CUTANEOUS LEISHMANIASIS IN GUINEA PIGS (Cavia porcellus)
}

\section{Roselene Ecco $^{1}$ Ingeborg Maria Langohr ${ }^{2}$ João Eduardo Wallau Schossler ${ }^{3}$ Severo Sales de Barros ${ }^{4}$ Claudio Severo Lombardo de Barros ${ }^{5}$}

\section{- RELATO DE CASO -}

RESUMO

\begin{abstract}
São descritos três casos de leishmaniose cutânea em cobaias (Cavia porcellus) machos de dois anos de idade que eram criadas como animais de estimação. As lesões macroscópicas eram semelhantes em duas cobaias e consistiam de nódulos cutâneos ulcerados no aspecto dorsal do pavilhão auricular de ambas as orelhas. Na outra cobaia, a lesão consistia de engrossamentos irregulares na pele do escroto que apareceram após uma orquiectomia realizada dois meses antes. A histopatologia era semelhante nos três casos e consistia de acentuada dermatite granulomatosa, com ulceração e numerosas formas protozoárias amastigotas no citoplasma de macrófagos, sugestivas de Leishmania sp. A microscopia eletrônica confirmou o diagnóstico de leishmaniose.
\end{abstract}

Palavras-chave: leishmaniose cutânea, cobaia, Cavia porcellus, Leishmania sp., doenças de animais de laboratório.

\section{SUMMARY}

Three cases of cutaneous leishmaniasis in three twoyear-old male guinea pigs (Cavia porcellus) raised as pets are described. Gross lesions were similar in two of the guinea pigs and consisted of raised and ulcerated cutaneous nodules in the dorsal aspect of the pinna of both ears. In another guinea pig the lesion consisted of irregular thickenning in the skin of the scrotum which followed an orchiectomy performed two months before. Histopathology was similar in the three cases and consisted of severe diffuse granulomatous dermatitis with ulceration and numerous intrahistiocytic protozoal amastigotes resembling Leishmania sp. Electron microscopy confirmed the diagnosis of leishmaniasis.

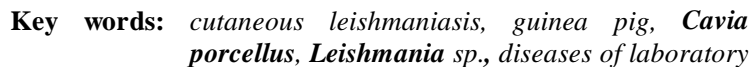
animals.

\section{INTRODUÇÃO}

A Leishmaniose é uma zoonose causada por um protozoário intracelular do gênero Leishmania (VAN DER LUGT \& STEWART, 1994) que tem seu ciclo biológico em dois hospedeiros, um vertebrado e um invertebrado (GENARO, 1995). Estudos eco-epidemiológicos na América Latina, particularmente no Brasil, reconheceram a presença de 20 espécies neotropicais de Leishmania, incluindo os subgêneros Leishmania e Viannia, 14 das quais são conhecidas por infectar o homem (LAINSON, 1997). Clínica e epidemiologicamente, há três formas de leishmaniose (GENARO,1995; JONES et al., 1996; ASHFORD, 1998). Cada forma é associada com espécies específicas de Leishmania: leishmaniose

\footnotetext{
${ }^{1}$ Médico veterinário, aluno do programa de Pós-graduação em Medicina Veterinária, área de concentração em patologia, Centro de Ciências Rurais, Universidade Federal de Santa Maria (UFSM).

${ }^{2}$ Aluno do Curso de Medicina Veterinária da UFSM. Bolsista (PIBIC/CNPq) no Departamento de Patologia da UFSM.

${ }^{3}$ Médico Veterinário, Professor Adjunto do Departamento de Clínica de Pequenos Animais da UFSM.

${ }^{4}$ Médico Veterinário, Professor Visitante da Faculdade de Veterinária da UFPel. Bolsista da FAPERGS.

${ }^{5}$ Médico Veterinário, Professor Tïtular do Departamento de Patologia da UFSM, 97105-900, Santa Maria, RS, Brasil. E-mail: cslb@sm.conex.com.br. Autor para correspondência.
} 
dermocutânea (L. tropica, L. major), leishmaniose visceral (L. donovani) e leishmaniose mucocutânea (L. braziliensis, L. mexicana). A forma cutânea localizada nas orelhas de cobaias foi descrita no Brasil e o agente etiológico classificado como $\boldsymbol{L}$. enriettii (MUNIZ \& MEDINA, 1948; MACHADO et al., 1994).

Doenças causadas por Leishmania spp. são transmitidas por insetos hematófagos que incluem os gêneros Lutzomyia (no Novo Mundo) e Phlebotomus (no Velho Mundo). Certas moscas, tais como a Stomoxys calcitrans, podem mecanicamente transmitir a infecção. Ao sugar o sangue, o inseto se torna contaminado pela ingestão de macrófagos infectados contendo amastigotas de Leishmania spp. No intestino do vetor, os macrófagos infectados liberam amastigotas que se transformam em promastigotas. Ao sugar novamente sangue, o inseto infecta mais um hospedeiro mamífero (VAN DER LUGT \& STEWART, 1994; JONES et al., 1996; ASHFORD, 1998). Relata-se a ocorrência de três casos de leishmaniose cutânea em cobaias nos municípios de Santa Maria e Itaara, RS.

\section{RELATO DOS CASOS}

Casos de leishmaniose cutânea foram diagnosticados em três cobaias machos com dois anos, criadas como animais de estimação. A cobaia 1 foi trazida pelo proprietário do município de Itaara, RS, devido a tumores que apareceram nas orelhas do animal. O município de Itaara é localizado na zona de serra, vizinho ao município de Santa Maria, na região central do Rio Grande do Sul. As cobaias 2 e 3 pertenciam a um grupo de cinco cobaias criadas numa mesma residência no município de Santa Maria, RS. A cobaia 2 tinha lesões nodulares cutâneas em ambas as orelhas, semelhantes às da cobaia 1 . A cobaia 3 teve lesões cutâneas no escroto, dois meses após ter sido submetido a uma orquiectomia. A ferida cirúrgica nunca cicatrizou. A não ser pelas lesões descritas, as três cobaias estavam clinicamente bem e foram submetidas à eutanásia e necropsiadas a pedido dos proprietários. Material colhido na necropsia dos três animais foi processado para histopatologia e microscopia eletrônica conforme TABONE \& ANDUJAR, 1980.

\section{RESULTADOS}

As lesões macroscópicas eram semelhantes nas cobaias 1 e 2 e consistiam de aumentos de volume no pavilhão auricular de ambas as orelhas. A

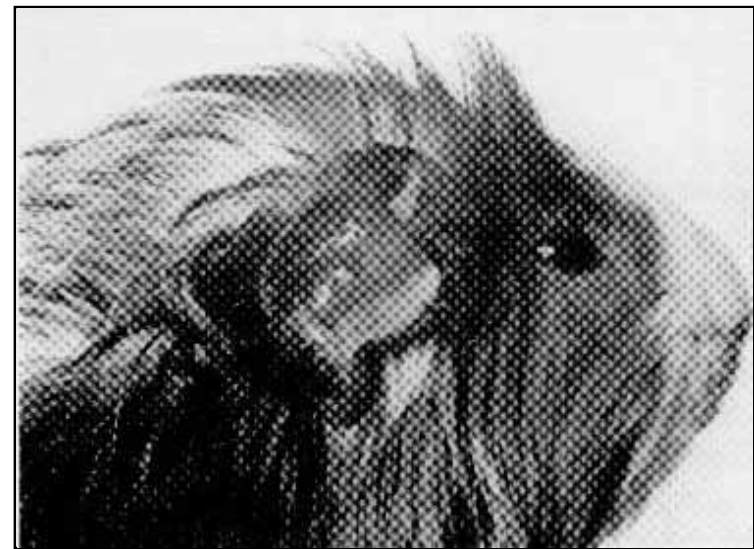

Figura 1 - Leishmaniose cutânea em cobaia. Dorso do pavilhão auricular tumefeito, brilhante, ulcerado e parcialmente recoberto por crostas.

pele da região dorsal do pavilhão era parcialmente alopécica, vermelho escura, brilhante, ulcerada e coberta por crostas (Figura 1). Ao corte, os nódulos tinham superfície branca, firme e homogênea. A cobaia 3 tinha a pele do escroto irregularmente espessada.

As alterações histológicas foram semelhantes em todos os casos e consistiam de infiltrado dérmico abundante e difuso de macrófagos, plasmócitos, linfócitos e moderada fibroplasia. Na lesão da cobaia 1, a infiltração por linfócitos era mais acentuada e na da cobaia 3 havia várias células gigantes multinucleadas. Numerosos macrófagos continham vacúolos parasitóforos contendo amastigotas com morfologia sugestiva de Leishmania sp. (Figura 2). Esses microrganismos eram ovóides, com 2-4 $\mu \mathrm{m}$ e

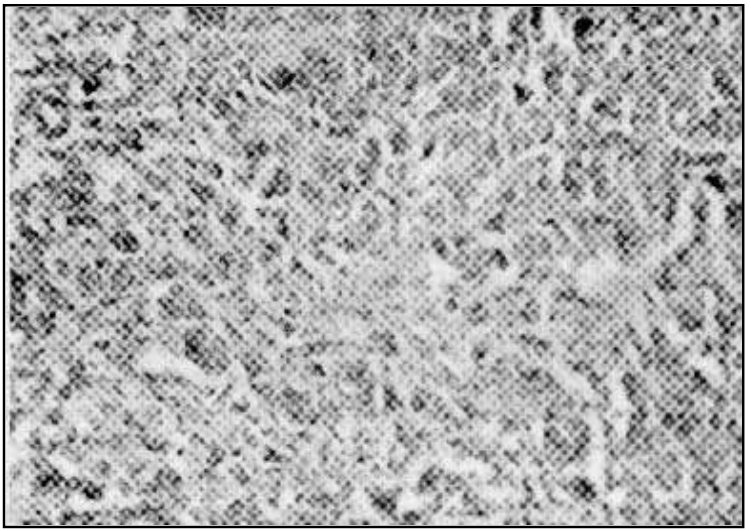

Figura 2 - Leishmaniose cutânea em cobaia. Histopatologia da lesão cutânea. Derme e tecido subcutâneo marcadamente infiltrados por macrófagos que contém vacúolos parasitóforos com amastigotas de Leishmania sp. Coloração de Hematoxilina e Eosina. 250 X. 
continham núcleo redondo e basofílico rodeado por halo claro. Nas lesões do cobaia 3, havia também numerosas amastigotas no interior das células gigantes multinucleadas. $\mathrm{O}$ exame ultra-estrutural revelou microorganismos protozoários consistentes com Leishmania sp. no interior de vacúolos parasitóforos intracitoplasmáticos (Figura 3).

\section{DISCUSSÃO}

Os casos de leishmaniose cutânea em cobaias foram diagnosticados pelos aspectos macroscópicos, histopatológicos e ultra-estruturais. A identificação da espécie não foi conseguida, mas casos anteriormente descritos de leishmaniose cutânea em cobaias são atribuídos a $\boldsymbol{L}$. enriettii (MEDINA, 1946; MUNIZ \& MEDINA, 1948; POULTER, 1979; MONROY et al., 1980; EL-ON et al., 1986; MACHADO et al., 1994; LAINSON, 1997).

Histoplasmose, toxoplasmose, blastomicose e tripanossomíase devem ser considerados no diagnóstico diferencial. Um quinetoplasto e um pequeno núcleo podem ser identificados em Leishmania spp., ao exame histopatológico, mas não nos agentes das três primeiras doenças. Trypanossoma cruzi apresenta quinetoplasto, mas a distribuição e o tipo das lesões na tripanossomíase

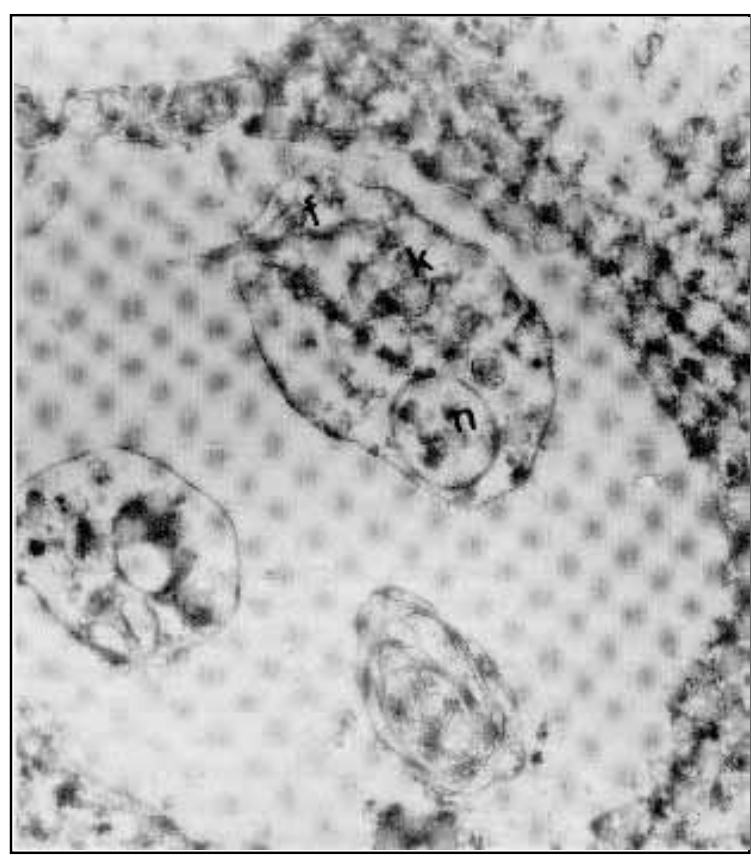

Figura 3 - Leishmaniose cutânea em cobaia. Microscopia eletrônica de espécimen de pele fixado em formol. Dois microrganismos (Leishmania sp.) podem ser observados. Num deles é possível identificar o núcleo $(\mathrm{N})$, o quinetoplasto (K) e resquícios do flagelo (F). 1800 $\mathrm{X}$. diferem dos da leishmaniose (VAN DER LUGT \& STEWART, 1994; JONES et al., 1996).

É interessante notar a predisposição para o desenvolvimento cutâneo das lesões da leishmaniose neste e em vários outros relatos de leishmaniose cutânea. A pele da face e das orelhas é mais exposta ao ambiente e pode predispor essas áreas à inoculação pelos vetores. Outra explicação para a freqüência nesses locais anatômicos pode estar relacionada à temperatura. $\mathrm{O}$ microrganismo que causa a forma visceral da leishmaniose cresce in vitro a $37^{\circ} \mathrm{C}$, enquanto os microrganismos que causam a doença mucocutânea crescem apenas a $34^{\circ} \mathrm{C}$. A ponta das orelhas é, provavelmente, mais fria que outras áreas da pele (COTRAN et $\boldsymbol{a l} .$, 1999).

O papel da doença como uma zoonose na região onde ocorreram os casos desse relato não foi ainda definido, mas deve ser investigado. Na região de Santa Maria e Itaara, não há relatos, até o momento, de leishmaniose cutânea em humanos residentes, mamíferos domésticos ou silvestres. É interessante notar, no entanto, que casos de leishmaniose visceral foram descritos em cães no município de Itaara (POCAI et al., 1998).

\section{REFERÊNCIAS BIBLIOGRÁFICAS}

ASHFORD, R.W. The leishmaniases. In: PALMER, S.R., SOULSBY, L., SIMPSON, D.I.H. Zoonoses. Oxford: Oxford University, 1998. Cap.43. p.527-543.

COTRAN, R.S., KUMAR V., COLLINS, T. Infectious diseases. In: Robbins pathologic basis of disease. $6 \mathrm{ed}$. Philadelphia: Saunders, 1999. p.391-392.

EL-ON, J., WITZTUM, A., SCHNUR, L.F. Protection of guinea pigs against cutaneous leishmaniasis by combined infection and chemotherapy. Infect Immun, v.51, n.1, p.704-706, 1986.

GENARO, O. Leishmaniose tegumentar. In: NEVES, D.P. Parasitologia humana. 8 ed. São Paulo : Atheneu, 1995. Cap.7. p.34-72.

JONES, T.C., HUNT, R.D., KING, N.W. Veterinary phathology. 6 ed. Baltimore : Williams \& Wilkins, 1996. Cap.12: Diseases due to protozoa: p.549-600.

LAINSON, R. On Leishmania enriettii and other enigmatic Leishmania species of the neotropics. Mem Inst Oswaldo Cruz, v.92, p.337-387, 1997.

MACHADO, M.I., MILDER, R.V., PACHECO, R.S., $\boldsymbol{e} t$ al. Naturally acquired infections with Leishmania enriettii Muniz and Medina 1948 in guinea-pigs from São Paulo, Brazil. Parasitology, v.109, p.135-138, 1994.

MEDINA, H.S.G. Estudos sobre leishmaniose. Primeiros casos de leishmaniose espontânea observada em cobaios. Arq Biol Tecnol, v.1, p.39-74, 1946. 
MONROY. A., RIDLEY, D.S., HEATHER, C.J., $\boldsymbol{e t}$ al. Histological studies of the elimination of Leishmania enriettii from skin lesions in the guinea-pig. Br J Exp Pathol, v.61, n.6, p.601-610, 1980

MUNIZ, J., MEDINA, H.S.G. Leishmaniose tegumentar do cobaio (Leishmania enriettii). Arq Biol Tecnol, v.3, art.2, p.13-35, 1948.

POCAI, E.A., FROZZA, L., HEADLEY, S.A., et al. Leishmaniose visceral (calazar). Cinco casos em cães de Santa Maria, Rio Grande do Sul, Brasil. Ciência Rural, v.28, n.3, p. 501-505, 1998 .
POULTER, L.W. The quantification of viable Leishmania enriettii from infected guinea-pig tissues. Clin Exp Immunol, v.36, n.1, p.24-29, 1979.

TABONE, E.J., ANDUJAR, M.R. Microscopia eletrônica de transmissão aplicada à biologia e patologia (fichas técnicas). Santa Maria : Universidade Federal de Santa Maria. 1980. 80p.

VAN DER LUGT, J.J., STEWART, C.G. Leishmaniasis. In: COETZER, J.A.W., THOMSON, G.R., TUSTIN, R.C. Infectious diseases of livestock - with special reference to Southern Africa. Oxford : Oxford University, 1994. v.1, cap.21. p.269-272

Ciência Rural, v. 30, n. 3, 2000. 